\title{
Hybrid Control of a Constrained Velocity Unicycle with Local Sensing
}

\author{
Apollon S. Oikonomopoulos, Savvas G. Loizou and Kostas J. Kyriakopoulos \\ Control Systems Lab, National Technical University of Athens \\ \{apoikos,kkyria\}@csl.mech.ntua.gr, sloizou@ieee.org
}

\begin{abstract}
This paper introduces a hybrid control scheme for steering a non-holonomic agent with limited sensing capabilities and input constraints through a stationary but unknown workspace, occupied by arbitrarily shaped obstacles. The considered constraints (i.e. constant linear and bounded angular velocities) apply to a wide category of vehicle systems. Limited sensing is realized by a radially bounded sensing device. The proposed hybrid controller respects the kinematic constraints while guaranteeing obstacle avoidance and convergence to a specified goal configuration. In addition to the analytical guarantees, we demonstrate the effectiveness of the proposed hybrid control scheme through non-trivial computer simulations.
\end{abstract}

\section{INTRODUCTION}

Autonomous mobile agents have attracted the interest of the control community during the last decades. Moreover, a particular category of mobile agents, the ones dealing with input-constrained non-holonomic systems, i.e. systems with bounded (or even fixed) linear velocity and bounded trajectory curvature, have been receiving increasing attention due to their wide application domain. These constraints are mostly imposed by operational considerations (i.e. improved fuel consumption, passenger comfort, etc.) rather than physical system capabilities and are encountered in numerous cases, including aircraft in cruising altitude, sea vessels and heavy vehicles. These kinematic constraints impose great restrictions on motion planning, that become especially important in the case of navigation in partially or completely unknown environments.

There are various efforts covering the issues of navigation in unknown spaces, as well as a multitude of works dealing with controlling non-holonomic agents with curvature constraints. Lumelsky and Stepanov [1] introduced a class of algorithms known as Bug algorithms for navigating unknown environments. Kamon et. al [2], [3] further extended these algorithms (DistBug, TangentBug, 3DBug) using range-sensor provided data, both in 2-dimensional and 3-dimensional environments. However, neither the original, nor the extended Bug algorithms take agents with aircraftlike kinematics into consideration. Loizou et. al [4] introduced a navigation function based approach to navigating partially known environments with guaranteed convergence. However this approach is applicable to holonomic agents only.

On the other hand, there is a variety of works on pathplanning for agents with aircraft-like kinematics. [5] proposes a two-phase 3D motion planner for fixed-wing UAVs that produces kinematically feasible paths in a world occupied by stationary obstacles, requiring global knowledge.
[6] treats obstacle avoidance in a limited scope, for circular obstacles only, within the proposed on-line path-planning algorithm. The path deformation method suggested in [7] is based on a partial a priori knowledge of the world and does not guarantee safety and convergence to the goal. [8] proposes a decentralized policy for conflict resolution in multi-agent systems with aircraft-like agents, but does not handle stationary obstacles.

In this paper we propose a hybrid motion-control scheme for agents with cruising-mode aircraft-like kinematics (planar motion) moving in a static but unknown environment with arbitrarily shaped obstacles. The agent has access to its posture $^{1}$ by means of a global positioning system but can only sense its surrounding environment by means of local sensing. The resulting hybrid controller respects the kinematic constraints while providing guaranteed properties of safety, stability and under some mild assumptions, completeness.

The remainder of this paper is structured as follows: section II presents preliminary notions and introduction to the problem. Section III discusses the sensing model thoroughly. Section IV presents the hybrid control strategy, while section $\mathrm{V}$ provides an analysis of the safety, convergence and completeness properties of the hybrid controller. Section VI presents simulation results and the paper concludes with section VII.

\section{System Description \& Problem Statement}

Consider a non-holonomic agent occupying a disc $R=$ $\left\{q \in \mathcal{W}:\|q-\mathbf{x}\| \leq r_{i}\right\}$ in the workspace $\mathcal{W} \subset \mathbb{R}^{2}$, where $\mathbf{x} \in \mathbb{R}^{2}$ is the center of the disc and $r_{i}$ its radius. We define the configuration space of the agent as $\mathcal{C} \subset \mathcal{W} \times(-\pi, \pi]$. The posture of the agent is defined as: $p \triangleq\left[\mathbf{x}^{T} \theta\right]^{T} \in \mathcal{C}$ where $\mathbf{x}=\left[\begin{array}{ll}x & y\end{array}\right]^{T}$ is the position of the agent and $\theta$ its orientation. The agent kinematics can be described by the following unicycle model:

$$
\dot{p}=\left[\begin{array}{ccc}
\cos \theta \sin \theta & 0
\end{array}\right]^{T} u+\left[\begin{array}{lll}
0 & 0 & 1
\end{array}\right]^{T} \omega
$$

along with the constraints:

$$
\begin{gathered}
u=u_{0}=\text { const }>0 \\
-u_{0} f_{\max } \leq \omega \leq u_{0} f_{\max }
\end{gathered}
$$

$f=\frac{\omega}{u_{0}}$ is the curvature of the agent's trajectory. Constraint (2b) yields the following minimum turning radius for the agent: $r_{\min }=\frac{1}{f_{\max }}$. We will also need three additional

\footnotetext{
${ }^{1}$ The term posture means "position and orientation" throughout the whole paper
} 
virtual non-negative states: $\tau \in T \subseteq \mathbb{R}_{0}^{+}$called timer, $\zeta \in \mathbb{R}$ called entry distance and $\eta \in \Omega=\left[-u_{0} f_{\max }, u_{0} f_{\max }\right]$ called entry angular velocity. Let $\mathcal{S} \subset 2^{\mathcal{W}}$ denote the sensing space. Then $S_{L}(p): p \in \mathcal{C} \rightarrow s \in \mathcal{S}$ is the local sensing function. Note that the agent also has access to its posture by means of global positioning with respect to the destination reference frame and compass information. Finally, we define a set of discrete states for the system and denote it with $\mathcal{D}$.

In order to present and study the proposed control strategy, we will use a hybrid system representation [9]. Hence our agent will be represented by the following hybrid system:

$$
\mathcal{R}=\left\{\mathcal{X}, \mathcal{X}_{0}, \mathcal{X}_{F}, F, E, D o m, G, R\right\}
$$

where $\mathcal{X}=\mathcal{D} \times\{\mathcal{C} \times T \times \mathbb{R} \times \Omega\}$ is the state space, $\mathcal{X}_{0} \subseteq \mathcal{X}$ is the set of initial states, $\mathcal{X}_{F} \subseteq \mathcal{X}$ is the set of final states, $F: \mathcal{X} \rightarrow T \mathcal{C}$ assigns to each discrete state $q \in \mathcal{D}$ a vector field $\omega=F(q, \cdot), E \subseteq \mathcal{D} \times \mathcal{D}$ is the set of discrete transitions, Dom $: \mathcal{D} \rightarrow 2^{\overline{\mathcal{C}}}$ assigns to each discrete state $q \in \mathcal{D}$ a set $\operatorname{Dom}(q) \subseteq \mathcal{C}$ called the domain, $G: E \rightarrow \mathcal{D} \times 2^{\mathcal{C}}$ assigns to $e=(q 1, q 2) \in E$ a guard of the form $\left\{q_{1}\right\} \times U, U \subseteq \operatorname{Dom}\left(q_{1}\right)$, and $R: E \rightarrow \mathcal{D} \times 2^{\mathcal{C}}$ assigns to $e=\left(q_{1}, q_{2}\right) \in E$ a reset of the form $q_{2} \times V$, $V \subseteq \operatorname{Dom}\left(q_{2}\right)$. The trajectories of $\mathcal{R}$ start in $(q, x) \in X_{0}$ and consist of discrete transitions in $\mathcal{D}$, the absolutely continuous evolutions in $\mathcal{C}$ governed by (1), and the (not necessarily continuous) evolution of the virtual states $\tau, \zeta$ and $\eta$ governed by $\dot{\tau}=1, \dot{\zeta}=0, \dot{\eta}=0$ respectively. The continuous vector field $\omega=F\left(q_{i}, \cdot\right)$ provides the control action to steer our system. In order to yield dynamically feasible trajectories, the controller should yield continuous input trajectories. The guard $G$ specifies a subset of the state space where a certain transition is enabled. Since the trajectories of our system are absolutely continuous in $\mathcal{W}$, the reset map $R$ will be the identity map in $\mathcal{W}$, while in $T$ every transition will result in zeroing the timer $\tau$. Finally we require that the hybrid system is non-blocking, i.e. from every state either a continuous evolution or a discrete transition is possible.

The problem that we are considering can thus be stated as follows:

"Given are the system (1) along with the constraints (2) and a static but unknown workspace $\mathcal{W}$. The system has knowledge of its current posture but can only sense its local surrounding environment. Assuming that the workspace is valid, design a (hybrid) control system that will steer the system from any valid initial configuration to the destination configuration, while avoiding collisions."

The valid workspace assumption implies that i) the obstacles are finite, ii) there exists a path between the start and the goal position that is at least $4 r_{\text {min }}+2 r_{i}+2 \delta$ wide at any point, where $\delta$ is a safety margin, and iii) there is an obstacle-free disc of radius $2 r_{\text {min }}+r_{i}+\delta$ around the destination.

The valid initial configuration assumption requires that there is an obstacle-free disc of radius $2 r_{\min }+r_{i}$ around the initial position.

We denote the initial configuration with $p_{0}=\left[\begin{array}{ll}\mathbf{x}_{0} & \theta_{0}\end{array}\right]^{T}=$

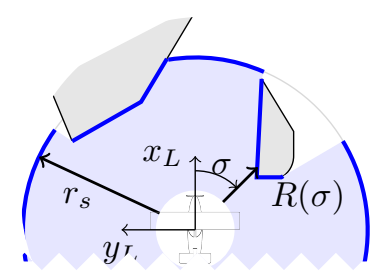

Fig. 1. The agent's sensing in operation. The segments in bold are returned as readings

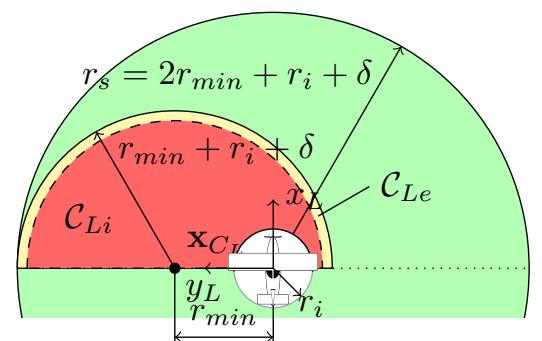

(a) The primary partitions defined on $D$.

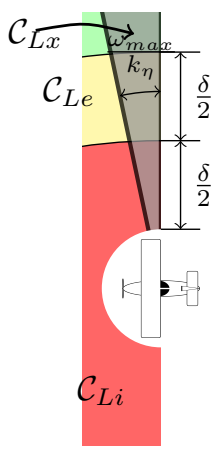

(b) Detail from figure 2(a)
Fig. 2. Partitions of the agent's sensing disc during a clockwise motion around an obstacle. The partitions for a counter-clockwise motion, denoted $\mathcal{C}_{R_{-}}$are mirrored wrt. $y_{L}$.

$\left[\begin{array}{lll}x_{0} & y_{0} & \theta_{0}\end{array}\right]^{T}$ and the destination configuration with $p_{d}=$ $\left[\begin{array}{ll}\mathbf{x}_{d} & \theta_{d}\end{array}\right]^{T}=\left[\begin{array}{lll}x_{d} & y_{d} & \theta_{d}\end{array}\right]^{T}$. For simplicity, we will assume $\mathbf{x}_{d}=\left[\begin{array}{ll}0 & 0\end{array}\right]^{T}$.

Also let $L$ be the local co-ordinate system of the agent, originating at the agent's center, with its $\mathrm{x}$-axis along the agent's longitudinal axis pointing forward according to the agent's motion and its y-axis extending to the left hand side. Figure 1 depicts the notation discussed. For the rest of this paper the notation $(x, y)_{L} \in S_{L}(p)$ shall denote a vector expressed in the agent's local co-ordinate system. The unit vector is denoted as $\hat{\mathbf{x}} \triangleq \frac{\mathbf{x}}{\|\mathbf{x}\|}$, a positive sign for angles and angular velocities shall indicate counter-clockwise revolution. The functions atan2, sign and sat used, are defined in the Appendix .

\section{LOCAL SENSING MODEL}

\section{A. Sensing}

The constraints affecting the maximum allowed trajectory curvature, $f_{\max }$, and the fixed linear velocity, $u_{0}$, lead to the possibility of infeasible initial configurations; a collision might be inevitable, since the agent cannot be steered arbitrarily fast to avoid the obstacle. This in turn implies that the minimal sensing capabilities that are required to ensure collision avoidance, are directly linked to the kinematic constraints.

We assume that the agent is equipped with a sonar-like sensing device (figure 1), capable of detecting the presence of objects within a radius $r_{s} \geq 2 r_{\text {min }}+r_{i}+\delta$. The sensing device scans radially and returns only the foremost outline of the objects detected, ignoring edges and any other objects 
in range that are masked by the foremost ones. We will now proceed to formulate this sensing device formally:

Let $Q_{j}$ be the set of points of $\mathcal{W}$ that are occupied by obstacle $j=1,2, \ldots, n$. We define the set of all obstacles, $\mathcal{Q} \triangleq \bigcup_{j=1}^{n} Q_{j}$. The obstacle-sensing device is modeled as a set of rays, originating at $(0,0)_{L}$. Each ray is modeled as a set, $S_{R}(\sigma) \triangleq\left\{r \mathbf{e}_{\sigma}: r \in\left(r_{i}, r_{s}\right]\right\}$, where $\mathbf{e}_{\sigma}$ is the unit vector in the direction $\sigma \in(-\pi, \pi]$ in the agent's local co-ordinate system. The reading for this ray is:

$$
R(\sigma) \triangleq \min _{\mathbf{q}_{L} \in S_{R}^{\prime}(\sigma)}\left\|\mathbf{q}_{L}\right\|, S_{R}^{\prime}(\sigma) \triangleq\left(\mathcal{Q} \cap S_{R}(\sigma)\right) \cup\left\{r_{s} \mathbf{e}_{\sigma}\right\}
$$

where $\mathbf{q}_{L}$ is in the agent's local co-ordinate system. Thus, every ray's reading is its closest wrt $(0,0)_{L}$ intersection point with any obstacle, or $r_{s}$ if it does not intersect with any obstacle. Finally, we model the sensor as the set of the readings of all rays covering a disc (figure 1) by means of the following local sensing function:

$$
S_{L}(p) \triangleq\{(\sigma, R(\sigma)): \sigma \in[-\pi, \pi]\}
$$

\section{B. Partitioning}

In order to exploit the data acquired through local sensing, we will now introduce a partitioning of the sensing area that is necessary for the development of our methodology.

Let $D$ be the half-disc defined by the sensing device's range in the agent's local coordinate system:

$$
D=\left\{\mathbf{q}_{L} \in \mathbb{R}_{L}^{2}:\left\|\mathbf{q}_{L}\right\| \leq r_{s} \wedge \mathbf{q}_{L}^{T}\left[\begin{array}{l}
1 \\
0
\end{array}\right] \geq 0\right\}
$$

Within the half-disc scanned by the sensing device, the following collision-critical zones are defined (figure 2):

$$
\begin{aligned}
\mathcal{C}_{X} & =\left\{y_{L} \in D:\left\|y_{L}-\mathbf{x}_{C_{X}}\right\|<r_{\text {min }}+r_{i}+\delta\right\} \\
\mathcal{C}_{X i} & =\left\{y_{L} \in D:\left\|y_{L}-\mathbf{x}_{C_{X}}\right\|<r_{\text {min }}+r_{i}+\frac{\delta}{2}\right\} \\
\mathcal{C}_{X e} & =\mathcal{C}_{X} \backslash \mathcal{C}_{X i} \\
\mathcal{C}_{L x} & =\left\{y_{L} \in D: \arg \left(y_{L}\right) \in\left[-\frac{\pi}{2},-\frac{\pi}{2}+\frac{\omega_{\max }}{k_{\eta}}\right]\right\} \\
\mathcal{C}_{R x} & =\left\{y_{L} \in D: \arg \left(y_{L}\right) \in\left[\frac{\pi}{2}-\frac{\omega_{\max }}{k_{\eta}}, \frac{\pi}{2}\right]\right\}
\end{aligned}
$$

where $X \in\{L, R\}, \mathbf{x}_{C_{L}}=\left[\begin{array}{ll}0 & r_{\min }\end{array}\right]_{L}^{T}$ and $\mathbf{x}_{C_{R}}=$ $\left[\begin{array}{ll}0 & -r_{\min }\end{array}\right]_{L}^{T}$, and $\frac{\omega_{\max }}{k_{\eta}}$ is a non-zero angle used to define a dead zone for obstacle avoidance (figure 2(b)). The parameter $k_{\eta}$ will be discussed later in this paper. Let $\mathcal{O}_{S}=\left\{[R(\sigma) \cos \sigma R(\sigma) \sin \sigma]^{T}:(\sigma, R(\sigma)) \in S_{L}(p)\right\}$ be the set of all points that correspond to the sensor's readings. We define $\mathcal{O}_{X x}=\mathcal{O}_{S} \cap \mathcal{C}_{X x}, \mathcal{O}_{Y}=\mathcal{O}_{Y i} \cup \mathcal{O}_{Y e}$, where $X x \in\{R i, R e, R x, L i, L e, L x\}$ and $Y \in\{R, L\}$.

\section{Hybrid CONTRol Strategy}

In this section we will define the parameters of the hybrid system (3). We assume that we have a valid workspace $\mathcal{W}$

\begin{tabular}{|c|c|c|}
\hline Transition & Guard & Reset \\
\hline $\mathrm{S}$ & $\hat{\mathbf{x}} \cdot \mathbf{e}_{\theta} \leq \mathbf{0} \wedge \tau \geq \frac{1}{\mathbf{k}_{\eta}}$ & $\tau:=0, \eta:=0$ \\
\hline $\mathrm{F}$ & $\hat{\mathbf{x}} \cdot \mathbf{e}_{\theta}>\mathbf{0} \wedge \tau \geq \frac{1}{\mathbf{k}_{\eta}}$ & $\tau:=0, \eta:=0$ \\
\hline $\mathrm{T}$ & $\tau \geq \frac{1}{k_{\eta}}$ & $\tau:=0$ \\
\hline Fs & $\hat{\mathbf{x}} \cdot \mathbf{e}_{\theta}>\mathbf{0} \wedge \tau \geq \frac{\mathbf{1}}{\mathbf{k}_{\eta}}$ & $\tau:=0, \eta:=\omega$ \\
\hline $\mathrm{Fl}$ & $\hat{\mathbf{x}} \cdot \mathbf{e}_{\theta}>\mathbf{0} \wedge\|\mathbf{x}\|<\zeta$ & $\tau:=0, \eta:=\omega$ \\
\hline Fr & $\hat{\mathbf{x}} \cdot \mathbf{e}_{\theta}>\mathbf{0} \wedge\|\mathbf{x}\|<\zeta$ & $\tau:=0, \eta:=\omega$ \\
\hline Dl & $\begin{array}{l}\hat{\dot{\mathbf{x}}} \cdot \hat{\mathbf{x}}=0 \wedge \mathcal{O}_{R}=\emptyset \wedge\|x\|< \\
\zeta\end{array}$ & $\tau:=0, \eta:=\omega$ \\
\hline Dr & $\begin{array}{l}\hat{\dot{\mathbf{x}}} \cdot \hat{\mathbf{x}}=0 \wedge \mathcal{O}_{L}=\emptyset \wedge\|x\|< \\
\zeta\end{array}$ & $\tau:=0, \eta:=\omega$ \\
\hline $\mathrm{L}$ & $\mathcal{O}_{L} \neq \emptyset \wedge \partial \mathcal{O}_{R e} \neq \emptyset$ & $\tau:=0, \zeta:=\|x\|, \eta:=\omega$ \\
\hline $\mathrm{R}$ & $\begin{array}{l}\left(\mathcal{O}_{R} \neq \emptyset \wedge \partial \mathcal{O}_{L e} \neq \emptyset\right) \vee \\
\left(\mathcal{O}_{R i} \cup \mathcal{O}_{L i}=\emptyset \wedge \partial \mathcal{O}_{L e} \neq\right. \\
\left.\emptyset \wedge \partial \mathcal{O}_{R e} \neq \emptyset\right)\end{array}$ & $\tau:=0, \zeta:=\|x\|, \eta:=\omega$ \\
\hline G & $\|x\|<\epsilon_{1} \wedge \hat{\mathbf{x}} \cdot \mathbf{e}_{\theta}<\mathbf{1}-\epsilon_{\mathbf{2}}$ & $\tau:=0, \eta:=\omega$ \\
\hline $\mathrm{E}$ & $\|x\|<\epsilon_{1} \wedge \hat{\mathbf{x}} \cdot \mathbf{e}_{\theta} \geq \mathbf{1}-\epsilon_{\mathbf{2}}$ & \\
\hline $\mathrm{Cr}$ & $\mathcal{O}_{L e} \backslash \mathcal{O}_{L x} \neq \emptyset \wedge \tau \geq \frac{1}{k_{\eta}}$ & $\tau:=0, \eta:=\omega$ \\
\hline $\mathrm{Wr}$ & $\mathcal{O}_{L e} \backslash \mathcal{O}_{L x}=\emptyset \wedge \tau \geq \frac{1}{k_{\eta}}$ & $\tau:=0, \eta:=\omega$ \\
\hline $\mathrm{Mr}$ & $\mathcal{O}_{R}=\emptyset \wedge \tau \geq \frac{1}{k_{\eta}}$ & $\tau:=0, \eta:=\omega$ \\
\hline $\mathrm{Cl}$ & $\mathcal{O}_{R e} \backslash \mathcal{O}_{R x} \neq \emptyset \wedge \tau \geq \frac{1}{k_{\eta}}$ & $\tau:=0, \eta:=\omega$ \\
\hline Wl & $\mathcal{O}_{R e} \backslash \mathcal{O}_{R x}=\emptyset \wedge \tau \geq \frac{1}{k_{\eta}}$ & $\tau:=0, \eta:=\omega$ \\
\hline Ml & $\mathcal{O}_{L}=\emptyset \wedge \tau \geq \frac{1}{k_{\eta}}$ & $\tau:=0, \eta:=\omega$ \\
\hline M & $\tau \geq \frac{r_{\min }+r_{i}}{u_{0}}$ & $\tau:=0, \eta:=\omega$ \\
\hline $\mathrm{N}$ & $\tau \geq \frac{3 \pi / 2}{\omega_{\max }}$ & $\tau:=0, \eta:=\omega$ \\
\hline
\end{tabular}
and valid initial configuration.

\section{A. Discrete States}

The hybrid system has 14 discrete states:

$$
\begin{array}{r}
\mathcal{D}=\left\{\mathbf{q}_{\mathbf{S}}, \mathbf{q}_{\mathbf{N}}, \mathbf{q}_{\mathrm{CR}}, \mathbf{q}_{\mathrm{R}}, \mathbf{q}_{\mathrm{DR}}, \mathbf{q}_{\mathrm{RR}}, \mathbf{q}_{\mathrm{FR}},\right. \\
\left.\mathbf{q C L}_{\mathbf{C L}}, \mathbf{q}_{\mathrm{L}}, \mathbf{q}_{\mathrm{DL}}, \mathbf{q}_{\mathrm{LL}}, \mathbf{q}_{\mathrm{FL}}, \mathbf{q}_{\mathrm{G}}, \mathbf{q}_{\mathrm{F}}\right\}
\end{array}
$$

These states have the following intuitive meaning:
TABLE I

THE HYBRID SYSTEM'S GUARDS AND RESETS

All transitions refer to figure 3

qs $\in X_{0}$ : Reorientation to face the destination. $\mathbf{q}_{\mathbf{N}} \in$ $X_{0}$ : Normal Motion in Free Space. $\mathbf{q C R}_{\mathbf{C R}} / \mathbf{q C L}_{\mathbf{C L}}$ CCW/CW Collision avoidance maneuver, $\mathbf{q}_{\mathbf{R}} / \mathbf{q}_{\mathbf{L}}$ : Boundary tracking with the obstacle's boundary on the RHS/LHS. qDR/qDL: Idle state. $\mathbf{q}_{\mathbf{R R}} / \mathbf{q}_{\mathbf{L} L}$ : $\mathrm{CCW} / \mathrm{CW}$ maneuver due to failure of RHS/LHS boundary tracking. $\mathbf{q G}_{\mathrm{G}}$ : Re-orientation at the destination. $\mathbf{q}_{\mathbf{F}} \in X_{F}$ : Final state. $\mathbf{q}_{\mathbf{F R}} / \mathbf{q}_{\mathbf{F L}}$ : Forced exit to the Free Space.

The set $E$ of possible transitions between the discrete states are depicted in the transition diagram in figure 3.

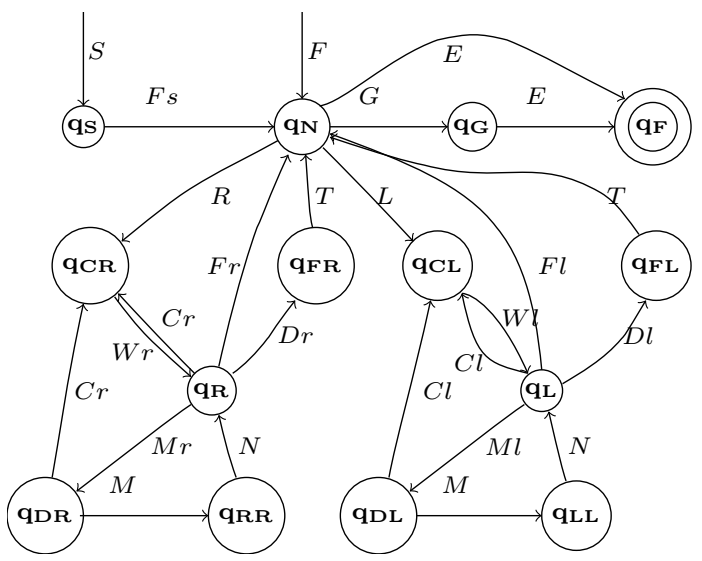

Fig. 3. The system's transition diagram. Transitions not shown on this diagram are ignored by the system.

${ }^{2}(\mathrm{C}) \mathrm{CW}=($ counter- $)$ clockwise, $\{\mathrm{L}, \mathrm{R}\} \mathrm{HS}=\{$ left-,right- $\}$ hand side 


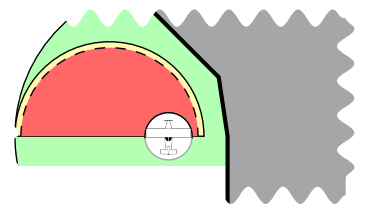

(a) Wall tracking $\left(\mathbf{q}_{\mathbf{R}}\right)$ or normal (b) Collision avoidance ( $\left.\mathbf{q C R}_{\mathbf{R}}\right)$. motion $\left(\mathbf{q}_{\mathbf{N}}\right)$.

Fig. 4. The use of the partitioned sensing data for state transitions

\section{B. Guards and resets}

The guards and the resets assigned to each transition are defined in Table I. An intuitive description of each guard condition follows:

F: The agent starts while the goal lies in front of it. S: The agent starts while it points away from the goal. $\mathbf{R}(\mathbf{L})$ An obstacle has been sensed on the agent's right (left) side's collision-critical zone. Fs: The goal lies in front of the agent and the agent has spent at least a finite amount of time, $T_{1}$ turning towards it. $\mathbf{F r}(\mathbf{F l})$ : The agent is avoiding the obstacle by tracing its border $\mathrm{CW}$ (CCW), has reduced its distance from the goal, the goal lies somewhere in front of it and no obstacles can be sensed between them. Dr (DI): The agent is avoiding the obstacle by tracing its border $\mathrm{CW}(\mathrm{CCW})$, has reduced its distance from the goal and has reached a local minimum of the distance-to-goal, $\|x\|$. G: The agent has come closer than $\epsilon_{1}$ to the goal, but its orientation its not the desired one. E: The agent has come closer than $\epsilon_{1}$ to the goal and its orientation is close to the desired orientation. $\mathbf{W r}(\mathbf{W l})$ : The agent moves along an obstacle that lies on its right (left) side and the agent's side opposite to the obstacle appears free of obstacles. $\mathbf{C r}(\mathbf{C l})$ : The agent is engaged in avoiding an obstacle that lies on its right (left) side and there appear to be obstacle parts in the opposite side. $\mathbf{M r}$ (MI): The agent has missed the obstacle boundary it was tracing from its sonar. M: The agent has spent time $T_{2}$ moving forward. $\mathbf{N}$ : The agent has spent time $T_{3}$ performing a circular maneuver.

\section{Vector fields}

Each discrete state is assigned a vector field $\omega=F(q, \cdot)$.

qs: $\omega=\omega_{\max } \operatorname{sat}\left(k_{\eta} \tau\right)$

$\mathbf{q N}_{\mathbf{N}}: \quad \omega=g(\eta, \tau)+\omega_{\max } \operatorname{sat}\left(k_{\eta} \tau\right) \operatorname{sat}(k \gamma)$

qCR: $\quad \omega=g(\eta, \tau)+\omega_{\max } \operatorname{sat}\left(k_{\eta} \tau\right)$

$\mathbf{q C L}_{\mathbf{C L}}: \quad \omega=g(\eta, \tau)-\omega_{\max } \operatorname{sat}\left(k_{\eta} \tau\right)$

$\mathbf{q}_{\mathbf{R}}: \quad \omega=g(\eta, \tau)-\operatorname{sat}\left(k_{\eta} \tau\right) \omega_{\max } \operatorname{sat}\left(k_{R}\left(d-d_{\text {nom }}\right)\right)$

$\mathbf{q}_{\mathbf{L}}: \quad \omega=g(\eta, \tau)+\operatorname{sat}\left(k_{\eta} \tau\right) \omega_{\max } \operatorname{sat}\left(k_{L}\left(d-d_{\text {nom }}\right)\right)$

$\mathbf{q}_{\mathbf{L L}}, \mathbf{q}_{\mathbf{F L}}: \quad \omega=g(\eta, \tau)-\omega_{\max } \operatorname{sat}\left(k_{\eta} \tau\right)$

qRR, $\mathbf{q}_{\mathbf{F R}}: \quad \omega=g(\eta, \tau)+\omega_{\max } \operatorname{sat}\left(k_{\eta} \tau\right)$

qDL, $\mathbf{q D R}_{\mathbf{D}}: \quad \omega=g(\eta, \tau)$

where $g(\eta, \tau)=\eta\left(1-\operatorname{sat}\left(k_{\eta} \tau\right)\right) . k_{\eta}$ is a tuning parameter controlling the "fade-in" of the vector field, ensuring that $\omega$ will be continuous in $t$. Its value is coupled with the agent's dynamic capabilities and should be adjusted as high as possible. The angle $\gamma$ is explained in figure 6. In the sequel, a relation connecting $k_{\eta}$ and $\delta$, the safety margin of the sensing device, will be given.

In almost every case, the vector field "smooths out" the initial (in the current state) angular velocity until the full saturated input is applied. The vector fields of states $\mathbf{q}_{\mathbf{R}}$ and $q_{L}$ modulate the input based on the difference of the actual and the desired distance from the obstacle's wall.

\section{Domains}

Each state has its own Domain of permissible continuous evolution

$$
\begin{aligned}
\operatorname{Dom}\left(\mathbf{q}_{\mathbf{S}}\right) & =\left\{p \in \mathcal{C}: \mathcal{O}_{L}=\mathcal{O}_{R}=\emptyset\right\} \\
\operatorname{Dom}\left(\mathbf{q}_{\mathbf{N}}\right) & =\left\{p \in \mathcal{C}: \hat{\mathbf{x}}^{T} \hat{\mathbf{x}}>0 \wedge\left(\mathcal{O}_{L} \neq \emptyset \oplus \mathcal{O}_{R} \neq \emptyset\right)\right\} \\
\operatorname{Dom}\left(\mathbf{q}_{\mathbf{C R}}\right) & =\operatorname{Dom}(\mathbf{q} \mathbf{C L})=\left\{p \in \mathcal{C}: \mathcal{O}_{R} \neq \emptyset \wedge \mathcal{O}_{L} \neq \emptyset\right\} \\
\operatorname{Dom}\left(\mathbf{q}_{\mathbf{R}}\right) & =\left\{p \in \mathcal{C}: \mathcal{O}_{R} \neq \emptyset \wedge \mathcal{O}_{L} \backslash \mathcal{O}_{L x}=\emptyset\right\} \\
\operatorname{Dom}\left(\mathbf{q}_{\mathbf{L}}\right) & =\left\{p \in \mathcal{C}: \mathcal{O}_{L} \neq \emptyset \wedge \mathcal{O}_{R} \backslash \mathcal{O}_{R x}=\emptyset\right\} \\
\operatorname{Dom}\left(\mathbf{q}_{\mathbf{X X}}\right) & =\operatorname{Dom}\left(\mathbf{q}_{\mathbf{F X}}\right)=\{p \in \mathcal{C}\} \\
\operatorname{Dom}\left(\mathbf{q}_{\mathbf{D L}}\right) & =\left\{p \in \mathcal{C}: \mathcal{O}_{R} \backslash \mathcal{O}_{R x}=\emptyset\right\} \\
\operatorname{Dom}\left(\mathbf{q}_{\mathbf{D R}}\right) & =\left\{p \in \mathcal{C}: \mathcal{O}_{L} \backslash \mathcal{O}_{L x}=\emptyset\right\}
\end{aligned}
$$

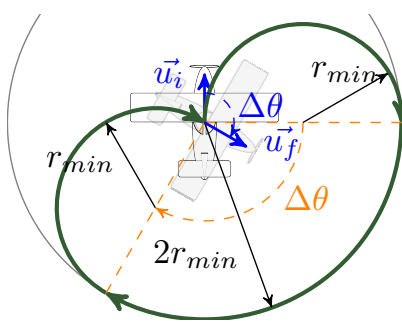

Fig. 5. Re-orientation maneuver of $\mathbf{q}_{\mathbf{G}}$. This maneuver can be performed inside a disc with radius $2 r_{\text {min }}+r_{i}+\delta$

\section{ANALYSIS OF THE HYBRID SySTEM}

We will now study the properties of the hybrid system related to safety convergence, completeness, constraint satisfaction and dynamic feasibility. We have the following regarding convergence and completeness:

Proposition 1: The trajectories of Hybrid System (3) for every valid workspace and valid initial configuration, converge in finite time to the destination configuration $p_{d}$.

Proof: Without loss of generality we will consider the destination configuration to be the origin, i.e. $p_{d}=$ $\left[0,0, \theta_{d}\right]^{T}$. Consider the following Lyapunov function $V_{N} \equiv$ $d=\sqrt{\mathbf{x}^{T} \mathbf{x}}$ that is assigned to the discrete state $q_{N}$. We can state the following:

Lemma 1: The trajectories of (3) while in $q_{N}$, satisfy $\dot{V}_{N}<0$.

Proof: While in $q_{N}$, the trajectories flow under the control law assigned to this state. No obstacles are being sensed between the agent and the goal. The objective of the control law of this state is to eliminate angle $\gamma$ (see figure 6). In $\operatorname{Dom}\left(q_{N}\right),|\gamma|<\frac{\pi}{2}$ is satisfied. Note that this angle is measured from the agent in a $\mathrm{CCW}$ fashion.(i.e. in figure 6 it has a negative sign). The rates of change of $\theta_{\delta}, d$ and $\gamma$ are:

$$
\begin{aligned}
\dot{\theta_{\delta}} & =\frac{u_{0}}{d} \sin \gamma \\
\dot{d} & =-u_{0} \cos (\gamma) \\
\dot{\gamma} & =\dot{\theta}_{\delta}-\dot{\theta}=\frac{u_{0}}{d} \sin \gamma-\omega
\end{aligned}
$$


Corollary 5.1: It holds that $\dot{d}(\tau)<0$, for $\tau \in\left[0, \frac{1}{k_{\eta}}\right]$

Proof: See appendix.

We will now study what happens for $\tau>\frac{1}{k_{\eta}}$. Assuming that $k \gamma>1$ and substituting the control law for $q_{N}$ in equation (6), we get:

$$
\dot{\gamma}=\dot{\theta}_{\delta}-\dot{\theta}=\frac{u_{0}}{d} \sin \gamma-\omega_{\max }
$$

Equation (7) expresses the rate of angular discrepancy that depends on both $\gamma$ and $d$. If this rate becomes non-negative, then the agent will not be able to converge to the goal. So, a lower bound $d \geq d_{\min }$ is sought, such that the agent will be able to converge in a neighborhood around the goal when starting from a distance $\|\mathbf{x}\|>d_{\min }$ with an arbitrary $\gamma \in\left(-\frac{p i}{2}, \frac{p i}{2}\right)$ while $k \gamma>1$. Without loss of generality we assume that $\gamma>0$, i.e. $\gamma \in\left(\frac{p i}{2}, 0\right]$. Assume that we had a slower controller capable only of performing:

$$
\omega=c+\frac{u_{0}}{d} \sin \gamma \leq \omega_{\max }
$$

Then it would be true that $\dot{\gamma}=-c$. Assuming that the agent enters $\mathbf{q}_{\mathbf{N}}$ at a distance $d_{0}$ from $\mathbf{x}_{G}$ with an angle $\gamma=\gamma_{0}$. Then, from (4), (5) and (8) follows that $d_{0} \geq \frac{u_{0}}{c}, c \leq$ $\frac{\omega_{\max }}{2}$. Thus the minimum distance $d_{0}$ from which the agent may converge to the goal from any admissible $\gamma$ is $d_{0_{\text {min }}}=$ $\frac{2}{f_{\max }}=2 r_{\min }$ for $c=\frac{\omega}{2}$. Thus we require that there is a disk: $D_{G}=\left\{\mathbf{q} \in \mathcal{W}:\left\|\mathbf{q}-\mathbf{x}_{G}\right\| \leq 2 r_{\min }+r_{i}+\delta\right\}$ such that $D_{G} \cap \mathcal{Q}=\emptyset$.

To summarize, it is possible to apply a control input that will bring $\gamma$ to zero in finite time. Since $\gamma$ remains in $\left(-\frac{p i}{2}, \frac{p i}{2}\right), \dot{d}<0$ while in $q_{N}$. Thus the agent continuously decreases its distance from the origin while in $q_{N}$.

Lemma 2: For a valid workspace, transitions $\mathrm{R}$ and $\mathrm{L}$ from $\mathbf{q}_{\mathbf{N}}$ result in a transition back to $\mathbf{q}_{\mathbf{N}}$ in finite time. Moreover the transition $q_{N} \stackrel{R / L}{\rightarrow} \ldots \stackrel{F}{\rightarrow} q_{N}$ results in a nonzero reduction of the level of the Lyapunov function $V_{N}$.

Proof: Let $\mathcal{C}$ be the set of all closed paths created during all possible transitions $q_{N} \stackrel{R / L}{\rightarrow} \ldots \stackrel{F}{\rightarrow} q_{N}$. Now take every close path from $\mathcal{C}$ and create it's Jordan equivalent that encloses the same obstacle(s) (i.e. throw away the portions of paths created by the transitions $q_{R / L} \stackrel{M r / M l}{\rightarrow} \ldots \stackrel{N}{\rightarrow} q_{R / L}$ ). Call this set $\mathcal{B}$. Let $g_{j}(s):[a, b) \subseteq \mathbb{R} \rightarrow \mathcal{W}$ be a parametric representation of any path in $\overline{\mathcal{B}}$, with $b-a=S$ the length of the path. $g_{j}(s)$ is by construction continuous and differentiable and required to be a bijection. Let $\mathcal{I}^{-}=\inf \mathcal{B}$ and $\mathcal{I}^{+}=\sup \mathcal{B}$. Let also $\nabla g_{j}(s)=\left[\frac{\partial g_{j}^{x}(s)}{\partial s} \frac{\partial g_{j}^{y}(s)}{\partial s}\right]^{T}$ be the vector tangent to the path at $g_{j}(s)$ and $\nabla^{\perp} g_{j}(s)$ be the vector perpendicular to $\nabla g_{j}(s)$, pointing towards $\mathcal{I}^{+}$.

With a reasoning similar to that of [4], we assert the following:

Lemma 3: $V_{N}$ attains a minimum along $g_{j}(s)$ at a point $\mathbf{h}_{\mathbf{b}_{\text {min }}}$.

Proof: Assume that $\|\mathbf{x}\|$ does not attain a minimum along $g_{j}(s)$. Then, since $\|\mathbf{x}\|$ is smooth and $g_{j}(s), s \in[a, b)$ is a bounded set the following has to be true: $\left\|g^{-1}(s)\right\|=$ const $\forall s \in[a, b)$. This means that the curve would have to be a circle, containing the origin at its center. This however would require the goal to be in $\mathcal{I}^{-}$, making the goal configuration infeasible. Thus, $\|x\|$ attains a minimum along $g_{j}(s)$ at $h_{b_{\text {min }}}$.

Lemma 4: The following relations hold:

$$
\begin{aligned}
& \hat{\mathbf{h}}_{b_{\text {min }}}^{T} \cdot \nabla g_{j}\left(g_{j}^{-1}\left(\mathbf{h}_{b_{\text {min }}}\right)\right)=0, \hat{\mathbf{h}}_{b_{\text {min }}^{T}}^{T} \cdot \nabla^{\perp} g_{j}\left(g_{j}^{-1}\left(\mathbf{h}_{b_{\text {min }}}\right)\right)<0 \\
& \quad \text { Proof: See [4] }
\end{aligned}
$$$$
\text { Proof: See [4] }
$$

According to Lemma 3 the agent will reach a point along the obstacle's surface where the Lyapunov function $V_{N}$ will be minimized. According to Lemma 4, at that point the obstacle will not lie between the agent and the origin. Thus the agent will never engage in obstacle avoidance at this point of minimum.

Lemma 5: Along $g_{j}(s)$ there is always a suitable exit point where the conditions for the transition $\mathbf{F r}(\mathbf{F l})$ are satisfied.

Proof: Let $\mathcal{L}_{e}$ be the level-set of $\|\cdot\|$ at which the entry-point to the obstacle, $\mathbf{x}_{e}$ belongs. Let $\mathcal{L}_{m}$ be the levelset of the minimum $h_{b_{\text {min }}}$ attained according to Lemma 3. Then there exists a segment of $g_{j}(s), \mathcal{S}_{e m}$ that has exactly one point in $\mathcal{L}_{e}, \mathbf{x}_{e}$ and one in $\mathcal{L}_{m}, \mathbf{x}_{m}$ and joins both level sets, the following is valid:

$$
\|\mathbf{x}\|>\left\|\mathbf{x}_{m}\right\|, \forall \mathbf{x} \in S_{e m} \backslash \mathbf{x}_{m}
$$

Since $g_{j}(s)$ is differentiable and has a continuous derivative $\nabla g_{j}(s)$ and (9) holds, then there is $s_{1} \neq g_{j}^{-1}\left(\mathbf{x}_{m}\right)$ such that $\nabla g_{j}(s)^{T} \cdot g_{j}^{-1}(s)<0 \forall s \in\left(s_{1}, g_{j}^{-1}\left(\mathbf{x}_{m}\right)\right)$. In other words, there is an area of $g_{j}(s)$ just before $\mathbf{x}_{m}$, where $\measuredangle\left(\nabla g_{j}(s),-\nabla \phi\left(g_{j}(s)\right)\right)<\frac{\pi}{2}$ while $\phi\left(g_{j}^{-1}(s)\right)<\phi\left(\mathbf{x}_{e}\right)$. So, all conditions of $\mathbf{F r}(\mathbf{F l})$ hold and the agent may exit the obstacle avoidance mode.

This implies that at the exit point we will have a lower level of the Lyapunov function. The minimum dwell establishes a finite reduction of the Lyapunov level.

This implies that $V_{N}$ will be reduced to $\epsilon_{1}$ in finite time, after which we will either have an accepting condition or a reorientation maneuver (state $q_{G}$ ) that will result in an accepting condition.

The last part of the proof concerns the completeness of the algorithm. We have the following:

Lemma 6: $\mathcal{I}^{+}$has at most $2 r_{\text {min }}+r_{i}+\delta$ minimum distance from the enclosed obstacle(s) and at least $2 r_{\min }+r_{i}+\delta$ minimum distance from any non-enclosed obstacle(s).

Proof: $\mathcal{I}^{+}$is produced by states $\mathbf{q}_{\mathbf{X}}$ and $\mathbf{q}_{\mathbf{C X}}, X \in$ $L, R$. Let $Q_{j}$ be the set of the points of an obstacle. Let $\mathcal{Q}^{-}$ be the set of all obstacles enclosed by $\mathcal{I}+$ and $\mathcal{Q}^{-}$be the set of all non-enclosed obstacles. Since $\mathcal{I}^{+}$is collision-free, $\left(Q_{j} \in \mathcal{Q}^{+}\right) \oplus\left(Q_{j} \in \mathcal{Q}^{-}\right)$holds, where $\oplus$ denotes exclusive disjunction. $\mathcal{I}^{+}$lies completely in $\operatorname{Dom}(\mathbf{q} \mathbf{X}) \cup \operatorname{Dom}(\mathbf{q} \mathbf{x R})$. This means: $\mathcal{O}_{X^{\prime}} \neq \emptyset \forall p \in \mathcal{I}^{+}$, where $X^{\prime}=R$ if $X=$ $L$ and vice versa. From the definition of $\mathcal{O}_{X^{\prime}}$ follows that $\forall p \in \mathcal{I}^{+} \exists q \in \mathcal{Q}^{-}:\|q-p\| \leq 2 r_{\text {min }}+r_{i}+\delta$. Obstacles belonging in $\mathcal{Q}^{+}$do not trigger collision avoidance. That means $\forall p \in \mathcal{I}^{+} \nexists q \in \mathcal{Q}^{+}:\|q-p\| \leq 2 r_{\text {min }}+r_{i}+\delta$. Thus, $\mathcal{I}^{+}$has clearance at least $2 r_{\text {min }}+r_{i}+\delta$ from every non-enclosed obstacle and at most $2 r_{\text {min }}+r_{i}+\delta$ from any enclosed obstacle. 
Corollary 5.2: If there exists path at least $4 r_{\min }+2 r_{i}+$ $2 \delta$ wide connecting the initial and destination configurations (valid workspace assumption), then the hybrid system will converge.

Proof: This is follows from Lemmas 5 and 6.

This completes the proof.

Proposition 2: The hybrid system 3 is safe

Proof: By the valid initial condition assumption we will either start in $q_{N}$ or $q_{S}$, sufficiently away from obstacles. Existence of obstacles in $C_{X e}$, will cause a transition to $q_{C X}$ that guarantees that obstacles will "slide" inside $C_{X e}$. This can be seen by considering that by the choice of $k_{\eta}$ only the second term of controller $q_{C X}$ will be active for $t>\frac{1}{k_{\eta}}$. This implies that the obstacle(s) will have penetrated less than $\delta / 2$ in $C_{X e}$ when they start moving on a circle centered at $\mathbf{x}_{C_{X}}$ and eventually the obstacles will "slide" to exit $C_{X e}$ without ever entering $C_{X i}$. State $q_{R}$ is safe since if for some reason the vector field defined in this state cannot follow the obstacle's boundary and keeps approaching it, the obstacle at some point will enter $C_{X e}$ and a transition to $q_{C X}$ will take place. State $q_{D X}$ will activate a transition to $q_{C X}$ whenever an obstacle enters $C_{X e}$ and state $q_{X X}$ is safe by construction since transition $\mathrm{M}$ occurred without obstacles in the interior of $C_{X e}$. Hence while in $q_{X X}$ obstacles in the worst case will penetrate $C_{X e}$ less that $\delta / 2$ and eventually will "slide" to exit $C_{X e}$. State $q_{G}$ is safe by the valid destination assumption.

\section{Simulation RESUlts}

To demonstrate the feasibility of the proposed methodology, we have setup a computer simulation. Following are the simulation results from three case studies.

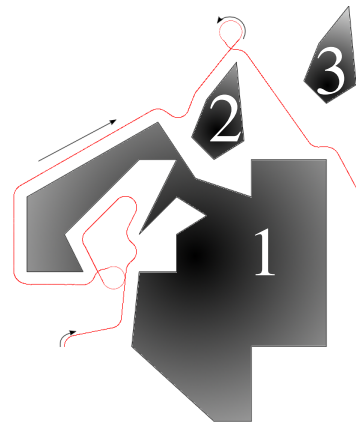

Fig. 7. Navigating around a complex non-convex obstacle

\section{Case study 1:}

This case study (figure 7) demonstrates the system's capability to track non-convex obstacles. Arrows indicate the motion direction. Notice that obstacles 1 and 2 are treated as one, since the gap between them is too narrow for the agent to escape from. Due to the limited sensing capability, the agent does not enter cavities it would possibly be unable to escape from, thus guaranteeing safety. When close to the destination, the agent performs a reorientation maneuver depicted in Fig. 5 to reach the goal orientation.
Case study 2:

The destination is positioned inside the convex hull of an obstacle (figure 8). Notice the "3-quarter" maneuver at the edge that makes it possible for the agent to enter the space enclosed by the obstacle. The reorientation maneuver at the goal, thus decoupling convergence to the goal and convergence to the desired orientation.

\section{CONCLUSIONS}

In this paper, a hybrid control scheme was introduced, for steering an agent with fixed velocity and bounded trajectory curvature through a stationary workspace occupied by arbitrarily complex stationary obstacles. The proposed controller guarantees collision avoidance and convergence to a set around the goal configuration, while generating continuous curvature trajectories with only local obstacle information. The methodology is scalable to very complex environments and analytical performance guarantees were provided. Current research directions include the incorporation of agent dynamics and extensions to scenarios with multiple agents.

\section{ACKNOWLEDGEMENTS}

The first and third author of this paper want to acknowledge the contribution of the European Commission through contract iFLY ("Safety, Complexity and Responsibility based design and validation of highly automated Air Traffic Management").

\section{APPENDIX}

This section contains definitions of some functions used throughout the paper:

$\operatorname{atan} 2(y, x) \triangleq \arg (x, y)$

$$
\operatorname{sign}(x) \triangleq\left\{\begin{array} { l l } 
{ - 1 , } & { x < 0 } \\
{ 1 , } & { x \geq 0 }
\end{array} \quad \text { sat } ( x ) \triangleq \left\{\begin{array}{ll}
-1, & x<-1 \\
x, & -1 \leq x \leq 1 \\
1, & x>1
\end{array}\right.\right.
$$

[1] V. J. Lumelsky and A. A. Stepanov, "Path-planning strategies for a point mobile automaton moving amidst unknown obstacles of arbitrary shape," in Algorithmica, vol. 2. Springer, 1987, pp. 403-430.

[2] I. Kamon and E. Rivlin, "Sensory-based motion planning with global proofs," in IEEE Transactions on Robotics and Automation, vol. 13, no. 6,1997 , pp. 814-821.

[3] I. Kamon, E. Rimon, and E. Rivlin, "Range-sensor based navigation in three dimensions," in Proceedings of the 1999 IEEE Int. Conf. on Robotics \& Automation. IEEE, 1999.

[4] S. G. Loizou, H. G. Tanner, V. Kumar, and K. J. Kyriakopoulos, "Closed loop motion planning and control for mobile robots in uncertain environments," in Proceedings of the 42nd IEEE Conf. on Decision and Control. IEEE, 2003.

[5] M. Hwangbo, J. Kuffner, and T. Kanade, "Efficient two-phase 3d motion planning for small fixed-wing uavs," in Proceedings of the 2007 IEEE Int. Conf. on Robotics \& Automation. IEEE, 2007.

[6] G. Ducard, K. C. Kulling, and H. P. Geering, "A simple and adaptive on-line path planning system for a uav," in Proceedings of the 15th IEEE Mediterranean Conf. on Control \& Automation. IEEE, 2005.

[7] O. Lefebvre, F. Lamiraux, C. Pradalier, and T. Fraichard, "Obstacles avoidance for car-like robots integration and experimentation on two robots," in Proceedings of the 2004 IEEE Int. Conf. on Robotics \& Automation.

[8] L. Pallottino, V. G. Scordio, E. Frazzoli, and A. Bicchi, "Probabilistic verification of a decentralized policy for conflict resolution in multiagent systems," in Proceedings of the 2006 IEEE Int. Conf. on Robotics and Automation. IEEE, 2006.

[9] G. J. Pappas, "Hybrid systems: Computation and abstraction," Ph.D dissertation, Univ. California at Berkeley, 1998. 